\title{
Des pratiques et espaces médicaux en transformation : effet générationnel ou conséquence de la féminisation de la profession?
}

Alice Denoyel-Jaumard et Audrey Bochaton

\section{OpenEdition}

Édition électronique

URL : http://journals.openedition.org/rfst/442

DOI : $10.4000 /$ rfst.442

ISSN : 2492-3672

Éditeur

Espaces et SOciétés (UMR 6590)

Référence électronique

Alice Denoyel-Jaumard et Audrey Bochaton, « Des pratiques et espaces médicaux en transformation effet générationnel ou conséquence de la féminisation de la profession ? », Revue francophone sur la santé et les territoires [En ligne], Genre, territoire et santé, mis en ligne le 21 décembre 2015, consulté le 06 avril 2021. URL : http://journals.openedition.org/rfst/442 ; DOI : https://doi.org/10.4000/rfst.442

Ce document a été généré automatiquement le 6 avril 2021.

La Revue francophone sur la santé et les territoires est mise à disposition selon les termes de la Licence Creative Commons Attribution - Pas d'Utilisation Commerciale - Partage dans les Mêmes Conditions 4.0 International. 


\title{
Des pratiques et espaces médicaux en transformation : effet générationnel ou conséquence de la féminisation de la profession?
}

\author{
Alice Denoyel-Jaumard et Audrey Bochaton
}

\section{Introduction}

1 Le processus de féminisation correspond à l'insertion d'un nombre croissant de femmes dans une activité qui a, pendant un temps, été uniquement exercée par des hommes (Cacouault-Bitaud, 2001). Au cours du 20 ème siècle, les progrès de la féminisation ont été considérables dans l'ensemble des sociétés occidentales. Ce phénomène a affecté les professions supérieures en raison de la levée progressive des barrières légales qui interdisaient jusqu'alors l'accès des femmes aux études supérieures conduisant à ces professions (Schweitzer, 2002). Dans les années 1880-1900, les femmes ont ainsi pu accéder aux cursus d'enseignement permettant de devenir enseignantes de lycée, médecins et avocates, ces professions étant perçues en continuité avec leur rôle de mère et leur garantissant une certaine flexibilité avec leur responsabilité domestique (Ibid). Si la féminisation du corps médical débuta à la fin du XIX e siècle, ce phénomène s'accéléra surtout à partir des années 1960. Ainsi, les femmes sont passées de 10\% des effectifs de médecins en 1960 à 33,4\% en 1982 (Lapeyre, 2006) et $44 \%$ en 2014 (Rault \& Le Breton-Lerouvillois, 2014). Selon les prévisions, leur proportion parmi les médecins en exercice devrait dépasser les 50\% en 2020 (Bessière, Breuil-Genier \& Darriné, 2004). Aujourd'hui le monde médical n'est donc plus exclusivement masculin comme il l'était initialement. Cependant des inégalités sexuées de carrière persistent, matérialisées par des femmes moins nombreuses à occuper des positions de pouvoir (Rosende, 2008). Ce phénomène, généralement connu sous l'expression "plafond de verre", n'est pas spécifique à la sphère médicale mais 
exprime plus généralement la disparition des femmes au fil de leur progression dans les hautes sphères professionnelles - libérales et salariées (Buscatto \& Marry, 2009).

2 La présence des femmes au cœur du corps médical a en outre toujours fait l'objet de représentations ambivalentes: d'un côté, la maternité et le "naturel doux et empathique" des femmes apparaissent comme des qualités incontestables au sein d'une profession qui consiste à prendre soin des autres; d'un autre côté néanmoins, exercer la médecine nécessite une force physique et mentale plus largement attribuée aux hommes. Aujourd'hui, alors que la médecine libérale est exercée à quasi parité, on peut s'interroger sur la manière dont progressent les représentations autour de la féminisation de la profession de médecins.

3 Dans le cadre de cet article, nous proposons d'examiner la question des représentations de cette féminisation au regard de l'accroissement des inégalités territoriales d'offre de soins. De nombreux rapports officiels et travaux scientifiques montrent que les écarts de répartition de médecins augmentent sur le territoire français et à différentes échelles (Maurey, 2013). La tendance actuelle est à la concentration des médecins en zone urbaine et par conséquent un délaissement des zones rurales désignées généralement par l'expression de "déserts médicaux ». Dans ce contexte, quels sont les facteurs évoqués et perçus pour expliquer ce phénomène? Dans quelle mesure la féminisation de la profession médicale est-elle instrumentalisée et désignée comme un déterminant central des évolutions actuelles de la médecine et de la distribution inégale des médecins sur le territoire français?

4 Outre la féminisation de la profession de médecins, il convient d'avoir à l'esprit que le secteur médical traverse également de nombreux changements liés au contexte actuel de départ massif à la retraite, à l'application du numerus clausus ${ }^{1}$ pendant de longues années et aux attentes de la nouvelle génération de médecins. Face à la diversité des facteurs démographiques, économiques, sociaux et territoriaux qui sont au cœur des changements de la profession, nous déterminerons la manière dont les représentations et les discours associés à la féminisation du métier perdurent et tendent à simplifier les évolutions complexes du métier.

\section{Croissance des inégalites territoriales en matière de médecine libérale}

5 Depuis la première convention médicale de 1971, la liberté d'installation des médecins libéraux est autorisée en France. Ce principe est toujours d'actualité aujourd'hui et les praticiens libéraux y sont très attachés.

\section{Une concentration urbaine au détriment des espaces ruraux}

6 Sur le territoire français, le nombre de médecins n'a cessé de progresser au cours de ces quarante dernières années. Après un quasi-triplement des effectifs entre 1968 et 1990, la progression a été légèrement plus modérée entre 2000 et aujourd'hui (Haas \& Vigneron, 2010), leur nombre s'élevant à 213227 en 2013 (Sicart, 2013). La France paraît donc dans une situation relativement favorable quant aux effectifs de praticiens. De plus, l'indicateur de desserte médicale ${ }^{2}$ montre que le pays compte environ 1 médecin pour 300 habitants, quand l'Organisation Mondiale de la Santé recommande 
une desserte d'un médecin pour 600 habitants (Picheral, 2001). Pourtant, si le nombre de médecins semble aujourd'hui suffisant au regard de la population, leur répartition sur le territoire français présente des inégalités manifestes.

7 Entre régions administratives, des inégalités importantes peuvent ainsi être observées. Si on examine la densité médicale ${ }^{3}$ on observe que celle-ci est nettement supérieure dans les régions méridionales françaises (notamment en Provence-Alpes-Côte-D’azur avec 408 médecins pour 100,000 habitants), mais aussi en région Ile-de-France qui compte 396 médecins pour 100,000 habitants (carte 1). Au contraire, la région Picardie affiche la densité médicale la plus faible (257 médecins pour 100,000 habitants), suivie de la région Centre (265 médecins pour 100,000 habitants).

Les inégalités sont encore plus marquées au sein des régions. La DREES précise que pour les médecins généralistes libéraux, $90 \%$ des inégalités de répartition s'observent entre les bassins de vie d'une même région, tandis que $10 \%$ des inégalités sont comptabilisées entre région (Fenina et al ., 2010)

Carte 1 : Densité médicale par région française en 2013

Densité de médecins (pour 100,000 hab)

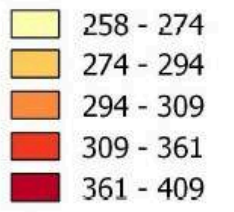

$0100200300 \mathrm{~km}$

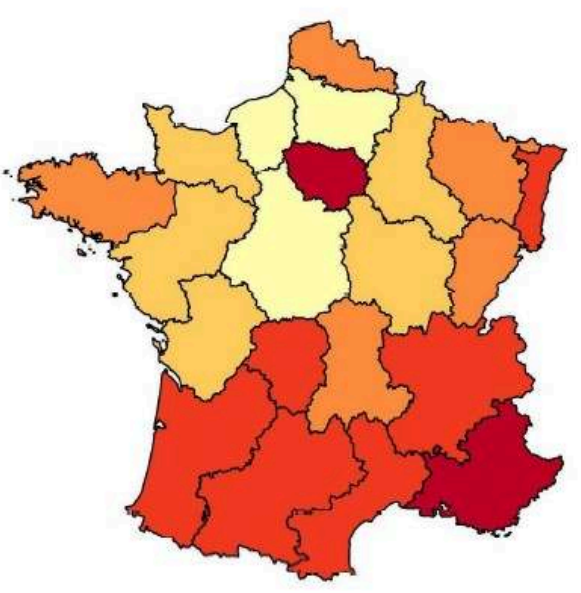

ह

Certains espaces infrarégionaux sont donc plus fortement délaissés par les praticiens que d'autres, et ils se caractérisent notamment par leur dominante rurale. Comme l'affirme Séverine Barbat-Bussière, "les différences en termes de services de santé entre ville et campagnes mettent ces dernières en situation d'infériorité " (BarbatBussière, 2009). En 2012, 20\% des médecins libéraux exerçaient ainsi dans des espaces à dominante rurale ${ }^{4}$ où résidaient néanmoins $27 \%$ de la population française. $80 \%$ des médecins libéraux se concentraient par conséquent en milieu urbain ${ }^{5}$.

\section{Au sein des villes, les centres urbains sont favorisés}

10 Si les espaces urbains trouvent grâce aux yeux des médecins en vue de leur installation, tous ne sont cependant pas attractifs de la même manière. Ainsi en 2007, alors que $14 \%$ de la population française résidait dans l'une des 646 Zones Urbaines Sensibles, ces dernières comptaient néanmoins $6 \%$ des effectifs médicaux et paramédicaux français (Cossardeaux, 2007). Par exemple, la Communauté d'Agglomération de Plaine 
Commune en Seine-Saint-Denis est confrontée à une fuite progressive des professionnels de santé, soit en raison des départs massifs à la retraite, soit à cause de l'insécurité « réelle, ressentie ou juste crainte » (Amat-Roze, 2011, 25). En 2011, à Stains et à Pierrefitte, $10 \%$ des médecins libéraux ont annoncé leur départ pour l'une de ces deux raisons. Or ces départs ne sont pas compensés par de nouvelles arrivées à cause de la mauvaise image de ces quartiers. En 2011, la Seine-Saint-Denis était le premier département en termes de déclarations de cas de violence sur médecins, puis le second derrière le département du Nord en 2012 et 2013 (Observatoire pour la Sécurité des Médecins, 2013). Certains médecins généralistes exerçant dans ces zones imputent leurs difficultés à trouver un successeur à la peur des nouvelles générations de venir s'installer dans des zones sensibles (Clavreul, 2011).

11 Ce processus de convergence vers les espaces urbains, et plus encore vers les centres villes, n'est pas spécifique à la profession médicale mais illustre plus globalement la concentration croissante des richesses, des emplois et des couches sociales les plus qualifiées dans les grandes agglomérations connu. Ce phénomène de métropolisation conduirait selon certains analystes à une dualisation de la société (Sassen, 1991) voire à des fractures socio-territoriales (Guilly 2010).

12 Les inégalités de répartition des médecins entre espaces urbains et espaces ruraux, et également au sein des espaces urbains, semblent concerner tout particulièrement les femmes-médecins, de plus en plus nombreuses dans la profession.

\section{L'installation préférentielle des femmes-médecins en milieu urbain}

13 Parmi certaines études sociologiques et démographiques, le constat est unanime : les femmes-médecins choisissent préférentiellement le milieu urbain pour l'installation de leur activité professionnelle. À partir d'une enquête menée auprès de 509 femmes médecins ayant été formées entre 1925 et 1950, il est apparu que ces dernières se sont installées "nettement plus fréquemment en milieu urbain (75\%) que leurs confrères. » (Paicheler, 2001, 188). Selon Daniel Sicart (2005), le taux de féminisation du corps médical est proportionnel à la taille de l'unité urbaine, et inversement pour les hommes. Ainsi, dans les villes comptant entre 200000 et 2 millions d'habitants, les femmes représentent $40,1 \%$ des effectifs, alors qu'en milieu rural, elles représentent $27,5 \%$ des médecins.

14 Nous avons confronté ces observations à une étude de cas sur les femmes-médecins généralistes de Bourgogne. En comparaison de la médecine libérale de spécialité, les femmes sont moins représentées dans la médecine libérale générale (43\% en 2014, contre $58 \%$ en gynécologie-obstétrique par exemple). Cependant, la part de femmes dans l'exercice de la médecine générale est croissante, ayant augmenté de 6 points entre 2007 et 2014 (Rault \& Le Breton-Lerouvillois 2014). En Bourgogne, au 1 er janvier 2013, 32\% des médecins généralistes libéraux étaient des femmes. Parmi elles, 91,6\% étaient installées dans l'espace à dominante urbaine, contre $89,9 \%$ des hommes et $89,6 \%$ de la population. Au sein de l'espace urbain, alors que $68 \%$ des omnipraticiens sont installés dans des pôles urbains (qui concentrent 52,2\% de la population), les femmes ont privilégié ce type d'installation dans $70 \%$ des cas, contre $67,2 \%$ des hommes (Tableau 1).

15 Il apparaît également que dans le cas d'une installation en pôle urbain, les femmesmédecins privilégient à $56,3 \%$ les pôles les plus peuplés contre $54,7 \%$ des hommes. Les 
femmes médecins généralistes libéraux de Bourgogne sont certes systématiquement surreprésentées dans l'espace urbain par rapport à leurs confrères masculins ${ }^{6}$ mais les différences ne sont pas significatives.

Tableau 1 : Répartition des médecins généralistes libéraux féminins et masculins en Bourgogne (au 1 er janvier 2013)

\begin{tabular}{|l|l|l|l|l|}
\hline & \multicolumn{1}{|c|}{ Effectifs } & \multicolumn{1}{|c|}{ Effectifs féminins } & Effectifs masculins & \multicolumn{1}{c|}{ Population } \\
\hline Espace urbain & $1047(90,5 \%)$ & $340(91,6 \%)$ & $707(89,9 \%)$ & $1470382(89,6 \%)$ \\
\hline Pôles urbains & $713(68,1 \%)$ & $238(70 \%)$ & $475(67,2 \%)$ & $767850(52,2 \%)$ \\
\hline $\begin{array}{l}\text { Couronnes } \\
\text { Périurbaines }\end{array}$ & $200(19,1 \%)$ & $66(19,4 \%)$ & $134(18,9 \%)$ & $423862(28,8 \%)$ \\
\hline $\begin{array}{l}\text { Communes } \\
\text { multipolarisées }\end{array}$ & $134(12,8 \%)$ & $36(10,6 \%)$ & $98(13,9 \%)$ & $278670(19 \%)$ \\
\hline Espace rural & $110(9,5 \%)$ & $31(8,4 \%)$ & $79(10,1 \%)$ & $171733(10,4 \%)$ \\
\hline Total & 1157 & 371 & 786 & 1642115 \\
\hline
\end{tabular}

Source : RPPS au 1 er janvier 2013, extraction ARS Bourgogne ; INSEE RGP 2010

16 A l'échelle intra-urbaine, les femmes-omnipraticiens ne se répartissent pas équitablement sur l'ensemble du territoire. En prenant l'exemple de Dijon, au 1 er janvier 2013, il apparaît que sur les 57 médecins généralistes libéraux installés dans un Quartier Politique de la Ville ${ }^{7}, 18$ (soit 31,5\%) sont des femmes. En comparaison, dans les autres quartiers du Grand Dijon, les femmes représentent $40,1 \%$ des effectifs d'omnipraticiens ${ }^{8}$.

17 La sous-représentation de femmes-médecins dans les espaces ruraux et les quartiers Politique de la Ville se confirme donc à l'échelle de la région Bourgogne mais les écarts observés entre les localisations des hommes et des femmes sont relativement modérés. Cependant, certains discours issus de la sphère médicale et relayés par les médias tendent à associer très étroitement l'accroissement actuel des inégalités territoriales de la médecine libérale et la féminisation du corps médical. Dans cette optique, les déserts médicaux s'expliqueraient ainsi en grande partie par les choix de vie des femmesmédecins et la spécificité de leur pratique médicale. Entre réalité et stéréotype, la suite du texte va démontrer comment ces discours s'ancrent dans une longue tradition de préjugés et véhiculent une image figée de la femme ainsi que de la profession de médecin.

\section{Discours et représentations autour de la profession de médecin et de sa féminisation}

Les femmes-médecins ont depuis toujours éveillé des représentations controversées dans un environnement qui fut, pendant des siècles, strictement masculin. Cette exclusivité masculine a fixé au fil du temps des normes quant aux attendus d'une «bonne» pratique médicale parmi lesquels l'exigence d'une disponibilité quasipermanente vis-à-vis du patient ou la nécessaire force physique pour exercer. "Dès lors, cette profession étant sous l'hégémonie masculine, il semblait évident que les médecins femmes devaient calquer leur comportement sur celui des hommes » (Deriaz, Bridel et al. 2010). Marlaine Cacouault-Bitaud (2001) a travaillé quant à elle sur la dévalorisation potentielle et symbolique de l'image de la profession médicale assimilée à la féminisation. La confrontation de ces normes professionnelles et de certaines 
représentations stéréotypées de la femme a créé au fil du temps des décalages entre les attendus imaginés d'une femme-médecin et la réalité. Cette situation explique et brouille jusqu'à aujourd'hui la compréhension de certaines évolutions récentes de la profession médicale comme la concentration accélérée de la médecine libérale dans les centres urbains.

\section{"Déserts médicaux, la faute aux femmes ?»}

Ce tweet du sénateur Mayet datant du 27 juillet 2015 peut certes paraitre anecdotique mais il livre néanmoins un aperçu des idées qui peuvent circuler sur la féminisation du corps médical en lien avec les difficultés actuelles de la profession. Les discours posant les femmes comme des responsables partielles de l'aggravation des inégalités de répartition des médecins entre le milieu urbain et le milieu rural deviennent de plus en plus fréquents à partir des années 1990 ; ce fut par exemple le cas en juillet 2013 lors d'un grand débat sur le désert médical organisé par l'Union française de la médecine libre en Lozère (Mouillot, 2013). Pour expliquer l'avancée du « désert médical » dans les zones rurales et "même dans de nombreuses zones urbaines ", l'UFML avance la féminisation de la profession comme une des raisons, "car les femmes médecins seraient peut-être plus sensibles à la recherche d'une meilleure qualité de vie familiale ». Ainsi, les femmes privilégient le temps extra-professionnel en choisissant des activités salariales surtout concentrées en ville, ou lorsqu'elles choisissent le libéral, privilégient des localisations urbaines. La féminisation allant croissante, cette problématique de l'inégale répartition des médecins devrait donc naturellement s'aggraver, portant à termes atteinte à la santé de la population. Nous pouvons ajouter qu'avec l'assignation de la femme-médecin comme responsable du "désert médical », le métier tout entier est stigmatisé : pour l'opinion, il ne respecte plus le serment d'Hippocrate ou le principe constitutionnel d'égalité d'accès aux soins, et préfère son confort à ses devoirs - ou pour la femme ses devoirs domestiques et maternels à ses devoirs professionnels. Il n'est donc plus le médecin dévoué et corvéable, à la limite du sacerdoce, auquel la famille était attachée.

Les liens établis aujourd'hui entre "désert médical » et «féminisation du corps médical » réaniment et actualisent des débats anciens sur la place et la légitimité des femmes médecins. Des stéréotypes en vigueur depuis des siècles comme, par exemple, la faiblesse physique de la femme à exercer la médecine, sont ainsi réactivés pour expliquer les préférences des femmes dans les choix de localisation.

\section{Portraits de femmes médecins : sorcières, faibles mais scrupuleuses}

21 L'exercice médical des femmes a suscité et suscitent encore pléthore de représentations. La femme-médecin de l'époque médiévale se résume au personnage de la sorcière. À partir du XIII e siècle, les femmes-médecins entrent dans l'illégalité car elles sont interdites d'enseignement au sein des facultés officielles, et sont pourchassées par l'Inquisition. Des bûchers sont ainsi levés jusqu'au XVIIe siècle pour supprimer les femmes soignantes, assimilées à des meurtrières (Dall'Ava-Santucci, 2004). Si les femmes-médecins ne sont plus perçues aujourd'hui comme des sorcières, force est de constater que la féminisation de la profession médicale suscite encore de 
nombreux préjugés quant à leur aptitude physique et leur engagement total pour exercer.

D'après les travaux historiques et sociologiques, la fragilité de la constitution féminine et leur "infériorité physique" ont été des arguments couramment avancés par les opposants à la féminisation pour justifier l'incapacité des femmes à exercer nombre de professions. Ainsi, selon l' Encyclopédie de Diderot et d'Alembert, le squelette féminin ne lui permet pas de marcher ou courir, et la femme est fréquemment atteinte de maladies de langueur. Ces arguments ont ensuite été réactivés à la fin du XIX ${ }^{e}$ siècle, au moment de l'introduction des premières femmes dans le cénacle médical. Il est alors affirmé que la force musculaire des femmes ne leur permet pas de soulever les malades, ou que leur période hebdomadaire menstruelle les rend physiologiquement fragiles (Nathalie Pigeard-Micaut, 2011). Des travaux récents de sociologues montrent que cette prétendue faiblesse physique des femmes est toujours d'actualité dans la culture de certaines spécialités médicales, comme celle de chirurgien. Ce métier, parmi les spécialités les moins féminisées, exigerait une "endurance physique ", "disposition socialement construite comme masculine » (Zolesio, 2009, 122).

Cet argument de la faiblesse féminine vient également justifier les discours sur une éventuelle prédisposition des femmes à s'installer dans les centres urbains réputés plus sécurisés.

« Allez au 15 è̀ M. (15 ème arrondissement de Marseille) faire une hospitalisation sur demande d'un tiers avec un gars qui jette tout son mobilier par la fenêtre comme je l'ai fait des nuits et des nuits à sos, est-ce que c'est la place d'une femme? Il n'y a pas de misogynie derrière ça mais, c'est d'ailleurs quand on est assez costaud en tant que mec on ne se sent pas trop à sa place, alors une petite minette qui arrive là-dedans. » (Témoignage d'un jeune médecin généraliste dans Hardy-Dubernet, 2005, 53)

Le regard porté par ce jeune médecin généraliste confronté à des accès de violence de certains patients, vient conforter l'idée d'un statut spécifique des médecins de sexe féminin et illustre la prégnance des représentations des femmes médecins au fil de l'histoire et jusqu'à aujourd'hui.

25 En écho à ce témoignage sur la problématique sécuritaire autour du métier de médecin, il est intéressant de noter que les médias télévisuels ont tendance à relayer plus fréquemment les atteintes aux médecins de sexe féminin alors même que la majorité des médecins victimes d'agressions sont de sexe masculin (en 2013, 55\% des agressions visaient des hommes). Le 27 mars 2012, un reportage du Magazine de la Santé (France 5) rapportant ces faits de violence faisait ainsi témoigner exclusivement des femmes-médecins. L'une d'elles, installée dans un quartier classé Zone Urbaine Sensible de la banlieue lyonnaise, témoignait avoir été victime de plusieurs agressions dont une tentative de viol. La discussion suivant le reportage avec la présidente de l'Association des Professionnels de santé de Pierrefitte, agressée 18 fois, fait des atteintes aux professionnels de santé dans les banlieues sensibles une "nouvelle mode» (Baudin, 2012). La presse écrite contribue également à relayer des faits d'agression exclusivement féminins, comme dans un article du Monde du 29 mars 2011, mentionnant le départ de la gynécologue et de l'ophtalmologue de Pierrefitte ainsi que l'agression d'une femme-médecin de Créteil. Ce matraquage médiatique tend à faire de la banlieue un territoire difficile pour la profession de médecins et qui plus est pour les femmes-médecins. Aussi, cette récurrence tend à faire oublier que les violences faites aux professionnels de santé sont aujourd'hui majoritairement verbales, et plutôt des 
violences psychologiques que physiques (Observatoire pour la Sécurité des Médecins, 2013).

26 A l'opposé de ces représentations négatives, les femmes sont des actrices majeures du care, qui revêt à la fois des activités et des attitudes qui relèvent de la sollicitude et du soin (Tronto, 1993). Les femmes sont alors réputées plus soucieuses, entre autres, de la santé d'autrui et plus disposées à faire preuve de douceur.

En 1987, Michelle Perrot définissait ce qui était entendu par «métier de femme », et expliquait ainsi la forte féminisation de professions de santé telle que celle d'infirmière. Les femmes ont historiquement été incluses dans la profession d'infirmière en raison des qualités nécessaires à ce métier, le plus souvent associées au sexe féminin. Ainsi, les infirmières et aides-soignantes ont d'abord été religieuses, puis des femmes louées pour leurs qualités matrimoniales et maternelles (Perrot, 1987). Cette approche répond à une conception du genre de type «essentialiste» ou «fixiste». Du fait de leurs caractéristiques sexuées naturelles et anatomiques (prétendue faiblesse physique, grossesses, allaitement...), les femmes se voient attribuer des qualités féminines qui vont avoir une transcription sociale et professionnelle. L'homologie entre sexe et genre est donc ici très nette (Le Feuvre, 2001), et les professions de santé sont de fait "socialement construites comme féminines» (Zolesio, 2009). Au contraire, pendant longtemps, la profession de médecin a nécessité des qualités "masculines» et "viriles », telles que l'autorité, la pugnacité, la force physique et mentale... Avec la féminisation des médecins, un glissement des qualités féminines jusqu'alors réservées à des professions telles que celle d'infirmière, s'est opéré vers la profession de médecin. La médecine deviendrait à son tour un " métier de femmes " (Hardy-Dubernet, 2005) : "Tout se passe comme si un déplacement des qualités professionnelles dévolues aux infirmières s'était opéré sur la personne du médecin " (Divay, 2006, 4). Les études relatives à la qualité supérieure des soins prodigués par les femmes-médecins se sont ainsi multipliées. En 2013, une étude québécoise fortement relayée par la presse française mettait par exemple en avant la bonne qualité des soins généralistes féminins, puisque les femmes passent plus de temps avec leurs patients et suivent de manière plus scrupuleuse les guides de bonnes pratiques (Borgès Da Silva et al., 2013). Ce discours, cette fois positif, part cependant lui-même d'un présupposé contestable de type essentialiste: les qualités propres aux femmes assurent la qualité des soins médicaux. Il permet cependant de contrebalancer les postulats et a priori tenus au sujet de la féminisation médicale, et notamment la dégradation du métier sur influence féminine.

\section{Médecin, mère, épouse : l'impossible « disponibilité permanente »}

Plusieurs études montrent que les femmes médecins travaillent en moyenne $34 \%$ de moins que leurs confrères masculins (Dormont et Samson, 2009). En outre, le temps partiel concernerait $25 \%$ des femmes médecins et seulement $2 \%$ des hommes ${ }^{9}$ (Simon \& Niel, 1999). Suivant ces raisonnements, nombreux sont les médecins à croire en l'équation " 2 femmes-médecins = 1 homme » en terme de temps de travail, alors que le nombre de femmes dans la profession augmente plus vite que le nombre de nouveaux médecins. À cela s'ajoutent les périodes de grossesse et de congés maternités, aussi incriminées dans la désorganisation des services de santé et dans la rupture de la continuité des soins (Divay, 2006). 

hommes fait écho plus globalement à la remise en question de l'ethos professionnel traditionnel évoqué par Lapeyre et Le Feuvre 2005 concernant la disponibilité permanente du médecin : " nous assistons à un changement de référentiel en matière d'organisation du travail ». Parmi les changements évoqués, des études montrent ainsi que les attentes des étudiantes en médecine sont en moyenne différentes de celles des étudiants et qu'elles inscrivent très tôt leur plan de carrière dans un contexte familial et social (Deriaz et al. 2010). En outre, la part du travail domestique est encore très majoritairement assurée par les femmes (Lapeyre et Le Feuvre, 2005) qui demeurent «bien plus que leurs confrères, placées dans l'obligation d'arbitrer au quotidien entre les contraintes de la vie familiale et celles d'une pratique professionnelle exigeante " (URML Rhône-Alpes, 2003, 7). Face à ce constat, l'évitement de spécialités médicales trop chronophages ou la préférence pour le secteur salarial plutôt que libéral favorisant la stabilité des horaires font par conséquent partie des choix privilégiés par une partie des femmes-médecins. Dans ce contexte, l'exercice en milieu rural peut apparaître moins attractif quand on sait que l'exercice médical dans cet environnement se caractérise par une surcharge de travail. Une étude de la DRESS de 2012 établit en effet que la durée hebdomadaire moyenne de travail des médecins généralistes en zone rurale s'élève à 60 heures en moyennes contre 56 heures en zone urbaine (Jakoubovitch et al., 2012). La comparaison des temps de consultations des femmes-médecins en fonction de leur lieu d'exercice montre donc une durée nettement plus courte dans les campagnes qu'en ville laissant supposer des consultations quotidiennes plus nombreuses (19,5 minutes contre 24,9 minutes en milieu urbain en Isère). Ces difficultés d'emplois du temps contraints s'observent également à travers les témoignages des professionnels de santé : $75 \%$ des femmes généralistes iséroises exerçant dans le rural estiment en effet être surchargées de travail contre seulement $11 \%$ en milieu urbain. Parmi leurs souhaits, il apparait une volonté de finir le soir à 17h30-18h et d'avoir plus de vacances (Edo, 2010). Ces contraintes de l'exercice médical en milieu rural, qui s'apparente à " un vrai sacerdoce » (Ibid, 79), s'expliquent par le nombre plus faible de médecins qu'en ville ainsi qu'à l'absence d'hôpitaux ou services d'urgence proches contraignant les médecins de 1ère ligne d'assurer la continuité des soins. Enfin, l'exercice en milieu rural se caractérise par des temps de trajet plus grands en raison de plus grandes distances à parcourir. peuvent conditionner une installation en milieu urbain à l'instar des choix faits par les femmes généralistes des villes d'Isère : $36,6 \%$ d'entre elles ont en effet tenu compte de l'emploi de leur conjoint tandis que $34,1 \%$ d'entre elles ont fait ce choix compte tenu de la souplesse des horaires et la scolarité des enfants (Ibid). Une enquête réalisée pour l'ensemble de la région Rhône-Alpes indique que respectivement $56,6 \%$ et $40,7 \%$ des femmes-médecins estiment que l'éducation des enfants et l'emploi de leur conjoint sont des contraintes fortes pour leurs choix professionnels (notamment de localisation), contre 21,7 et $10,4 \%$ des hommes (URML Rhône-Alpes, 2003, 67). La préférence des femmes pour un exercice en milieu urbain ne doit donc pas être pensée strictement à l'échelle individuelle mais également à l'échelle du couple puisque «l'évolution des rapports de genre ne va pas jusqu'à rendre normal que leur époux ou compagnon s'estime valorisé par un statut de conjoint de médecin ou un exercice professionnel d'appoint, comme ce fut longtemps le cas pour les femmes de médecin » (Bloy, 2013, 78). Lieu de concentration des activités et des services, l'espace urbain apparaît enfin

Revue francophone sur la santé et les territoires, Genre, territoire et santé 
comme un environnement favorable pour l'éducation des enfants, les services de garde, les loisirs, etc.

31 Le tropisme urbain féminin reflète des décisions certes individuelles mais fortement contraintes par l'organisation de la société française qui limite finalement le choix territorial féminin (Haas \& Vigneron, 2010) et l'oriente plus favorablement vers l'espace à dominante urbaine

\section{Au delà d'une analyse dichotomique : changements sociaux et effet génération}

32 Pour comprendre les logiques qui sous-tendent l'apparition des déserts médicaux et le renforcement des inégalités territoriales d'offre de soins, il convient de dépasser les discours et les explications genrés pour aborder plus largement les déterminants sociaux et économiques qui participent actuellement de ce phénomène. Comme le formule le syndicat des médecins libéraux, "il ne faudrait pas confondre "désert médical » avec féminisation médicale » ( http://www.lesml.org ); mais cela nécessite de s'extraire de la pensée dichotomique masculin/féminin et préférer l'image d'un continuum (voir l'entretien de Claire Hancock dans ce numéro) plus appropriée pour comprendre les évolutions communes à la profession de médecin et saisir les influences réciproques des médecins hommes et femmes, aînés et nouvelle génération, etc. Dans cette optique, les observations de Nathalie Lapeyre et Nicky le Feuvre (2005) sont très intéressantes et établissent que l'influence de la féminisation du corps médical va bien au-delà de la seule présence numérique des femmes: " avec l'arrivée des femmes sur le marché de l'emploi, les impératifs liés à la sphère privée tendent à s'immiscer dans les interstices des temporalités professionnelles de l'ensemble des médecins, hommes et femmes ». Dans ses travaux sur les "parcours féminins et masculins de spécialisation en médecine" réalisés en Suisse, M. Rosende (2008) remarque également l'influence du projet privé dans le projet professionnel des médecins, hommes et femmes confondus, ce qui l'amène à interroger plus largement l'émergence de nouveaux rapports au travail et à la carrière notamment chez les jeunes médecins.

33 Ainsi, les nouvelles générations souhaitent préserver du temps pour la famille ou d'autres activités sociales et tant les femmes que les hommes revoient profondément leur rapport au travail (Deriaz et al. 2010). Selon une enquête menée par le syndicat des internes de médecine générale (ISNAR-IMG) en 2011, les hommes comme les femmes estimaient les écoles comme le premier service nécessaire à côté de leur cabinet d'exercice, et l'écart entre les deux sexes s'avérait minime ( $72 \%$ des femmes et $70 \%$ des hommes). De manière similaire, des travaux ont montré une tendance au rapprochement des temps de travail des hommes et des femmes médecins au cours des années 90 (Simon et Niel, 1999).

En 2003, Robert Hérin affirmait déjà à propos de l'offre de soins libérale en BasseNormandie, que les nouvelles générations de médecins (sans distinction de sexe) se posent « des questions relatives au cadre de vie, les possibilités de scolarisation pour les enfants, l'éventail des emplois pour le conjoint, les qualités de l'environnement culturel et social, autant d'aspirations pour lesquelles les milieux ruraux sont désavantagés (...), cela d'autant plus qu'ils sont jeunes » (Hérin, 2003, 193). 
35 Tous ces éléments orientent donc les choix des jeunes médecins vers un exercice de la médecine dans un environnement de préférence urbain ce qui permet de penser les inégalités d'offre actuelle comme une question générationnelle et pas uniquement une question genrée.

36 Pour certains médecins, le problème est même plus profond puisqu'ils associent le processus de désertification médicale plus largement à la perte d'attractivité de l'exercice libéral de la médecine générale du fait des nombreuses contraintes liées à ce statut (charge administrative, horaires variables, etc.).

37 «L'hôpital public est [ainsi] devenu le premier employeur de généralistes » (Juilhard JM, 2007) ce qui aggrave d'autant plus les inégalités territoriales que les centres hospitaliers sont encore plus inégalement répartis sur le territoire que les médecins libéraux. L'exercice groupé dans le cadre de maisons de santé pluridisciplinaires (MSP) apparaît cependant comme un modèle en pleine ascension, car il permet de diminuer les contraintes administratives, d'assurer le partage des frais de secrétariat mais aussi un partage des informations sur les patients et donc un suivi facilité.

\section{Conclusion}

38 «Au lieu d'ignorer ou de stigmatiser les prétendues «spécificités féminines » en matière d'exercice médical, les pouvoirs publics et les instances de décision de la profession auraient intérêt à réfléchir davantage sur les effets de la dynamique du genre sur les aspirations de l'ensemble des médecins - hommes et femmes - quant aux conditions d'exercice de leur profession à l'avenir " (Lapeyre et Nicky le Feuvre 2005, p. 77). Dix ans après, cette préconisation reste d'actualité. Puisque les nouvelles générations de médecins investissent de plus en plus leur vie sociale et familiale, il semble indispensable aujourd'hui de repenser les modes d'exercice, les conditions de travail des médecins en lien avec leurs aspirations de vie. Quelle formule peut ainsi permettre de concilier vie privée et vie professionnelle tout en corrigeant les inégalités territoriales d'offres de soins? Certaines mesures adoptées par les pouvoirs publics ont visé notamment l'installation et le soutien des médecins exerçant dans des zones déficitaires : ces expériences sous forme d'incitation financière, contrat d'engagement pour les étudiants en médecine, incitations fiscales, aides des collectivités locales n'ont toutefois pas apporté de résultats notoires au cours des dernières décennies. Une revue de la littérature relatant des expériences internationales (Bourgueil, Mousquès et al., 2006) relativise en effet l'efficacité des mesures d'incitations financières par rapport aux caractéristiques intrinsèques du territoire choisi: conditions de vie, niveau d'équipement, contexte socio-économique, etc (Barnay et al. 2007). Dans ce contexte, comment impulser des changements en mobilisant la notion de territoire ? Il n'est en effet plus concevable aujourd'hui d'inciter individuellement les médecins à exercer ici ou là ; il convient plutôt de réfléchir plus étroitement aux conditions de l'attractivité des territoires, là où justement les aspirations de vie des uns et des autres prennent forme. Les inégalités territoriales d'accès aux soins et l'existence de zones déficitaires exigent donc une intervention plus consistante des géographes et des aménageurs. Compte tenu des nouvelles aspirations des jeunes médecins, les incitations à s'installer dans les zones sous-médicalisées devront certes porter sur les aménagements du travail (remplacement des médecins, exercice regroupé dans le cadre de maisons de santé) mais elles ne peuvent se limiter à la sphère professionnelle. Rendre un territoire 
attractif pour des médecins, c'est rendre ce territoire attractif pour tout un chacun. Cela exige de réinvestir plus globalement et dans différents secteurs les territoires à la marge, qu'ils s'agissent des campagnes et des banlieues déshéritées, et de modifier favorablement les conditions de vie localement.

Des réformes apparaissent urgentes et nécessaires dans le contexte actuel de baisse de la démographie médicale, baisse qui va continuer de diminuer jusqu'à 2025 avec une baisse de près de $10 \%$ des effectifs de médecins. D'ici là, des alternatives se mettent en place pour pallier les besoins de zones déficitaires; le recours à des médecins diplômés à l'étranger (principalement des pays de l'Europe de l'Est et du Maghreb) qui comptent en 2012 pour $27 \%$ des nouveaux médecins inscrits. Un article paru dans le Courrier International (avril 2015) relate aussi les migrations moins connus de médecins hollandais et belges dans les campagnes françaises par l'intermédiaire de cabinet de recrutement (entre 70 et 80 médecins nouvellement installés depuis 2007). Néanmoins, qu'il s'agisse d'un médecin étranger ou d'un médecin diplômé d'une ville française, la difficulté de créer des attaches et un environnement familier dans un lieu d'exercice nouveau reste la même.

Sans réforme profonde de la médecine libérale, une dernière alternative consistera plus simplement à remettre en cause la liberté d'installation des médecins comme cela est déjà le cas dans de nombreux pays d'Europe. Mais cette solution contraignante risque d'être complexe à mettre en œuvre du fait de la variété des situations et spécialités médicales et de l'attachement des médecins libéraux à leur liberté d'exercice.

\section{BIBLIOGRAPHIE}

AMAT-ROZE Jeanne-Marie, 2011, La territorialisation de la santé : quand le territoire fait débat, Hérodote, $\mathrm{n}^{\circ}$ 143, pp.13-32.

BARBAT-BUSSIERE Séverine, 2009, L'offre de soins en milieu rural. L'exemple d'une recherche appliquée en Auvergne, Clermont-Ferrand : Presses Universitaires Blaise Pascal.

BARNAY Thomas, HARTMANN L, ULMANN P, 2007, Réforme du " médecin traitant » et nouveaux enjeux de la médecine de ville en France, Revue française des affaires sociales 2007/1 (n $\left.{ }^{\circ} 1\right)$, p. 109-126.

BAUDIN Auriane, 2012, Insécurité des médecins, peut mieux faire, France5.fr et Allodocteurs.fr, [en ligne] http://www.allodocteurs.fr/actualite-sante-insecurite-des-medecins-peut-mieuxfaire-6581.asp?1=1 (02/09/2014).

BESSIERE Sabine, BREUIL-GENIER Pascale, DARRINE Serge, 2004, La démographie médicale à l'horizon 2025 : une actualisation des projections au niveau national, Drees Etudes et résultats, $\mathrm{n}^{\circ}$ 352, [en ligne] http://www.drees.sante.gouv.fr/IMG/pdf/er352.pdf (02/09/2014).

BLOY Géraldine, 2010, Entretien par Serge CANNASSE, Carnets de Santé, [en ligne] http:// www.carnetsdesante.fr/Bloy-Geraldine (02/09/2014). 
BLOY Géraldine, 2013, Jeunes généralistes et déserts médicaux. Un regard sociologique éclairant, Pratiques, Les cahiers de la médecine utopique, $\mathrm{n}^{\circ} 60, \mathrm{pp} .77-80$.

BORGES DA SILVA Roxane, MARTEL V., BLAIS R., 2013, Qualité et productivité dans les groupes de médecine de famille : qui sont les meilleurs? Les hommes ou les femmes?, Revue d'Épidémiologie et de Santé Publique, vol. 61, Suppl. 4, pp. S210-S211.

BOURDIEU Pierre, 1979, La distinction. Critique sociale du jugement, Paris : Editions de Minuit, coll. Le Sens commun.

BUSCATTO Marie, MARRY Catherine, 2009, « Le plafond de verre dans tous ses éclats ». La féminisation des professions supérieures au XX e siècle, Sociologie du travail, 51 (2009), 170-182.

CACOUAULT-BITAUD Marlaine, 2001, La féminisation d'une profession est-elle le signe d'une baisse de prestige ?, Travail, Genre et Sociétés, $n^{\circ}$ 5, pp. 93-115.

CLAVREUL Laetitia, 2011, 29 mars, Des médecins de plus en plus agressés, surtout verbalement, Le Monde [en ligne] http://www.lemonde.fr/societe/article/2011/03/29/des-medecins-de-plusen-plus-agresses-surtout-verbalement_1499961_3224.html (02/09/2014).

CLAVREUL Laetitia, 2011, 30 décembre, En Seine Saint-Denis, le blues de praticiens qui partent à la retraite sans successeur, Le Monde [en ligne] http://www.lemonde.fr/societe/article/ 2011/12/30/en-seine-saint-denis-le-blues-de-praticiens-qui-partent-a-la-retraite-sanssuccesseur_1624257_3224.html (02/09/2014).

COSSARDEAUX Joël, 2007, 29 mai, L'offre de soins se révèle sous-dimensionnée dans les cités, Les Echos n ${ }^{\circ}$ 19927, p. 5.

COUFFINHAL Agnès, LUCAS-GABRIELLI Véronique, MOUSQUES Julien, 2002, Où s'installent les nouveaux omnipraticiens? Une enquête cantonale sur 1998-2001, CREDES Questions d'Economie de la Santé, $\mathrm{n}^{\circ} 61$ [en ligne] http://www.irdes.fr/Publications/Qes/Qes61.pdf (02/09/2014).

DALL'AVA-SANTUCCI Josette, 2004, Des sorcières aux mandarines, Histoire des femmes médecins, Paris : Calmann-Lévy.

DENEFLE Sylvette (dir.), 2004, Femmes et villes. Tours : Maison des Sciences de l'homme «Villes et territoires ", Presses universitaires François-Rabelais.

DIVAY Sophie, 2006, Incidences de la féminisation de la profession de médecin en France sur le rapport au travail des étudiant-e-s et des jeunes généralistes, Communication présentée au Colloque international Travail-Emploi-Formation, quelle égalité entre les hommes et les femmes ? du CLERSE, 23-24 novembre 2006, Lille, [en ligne] http://halshs.archives-ouvertes.fr/ docs/00/27/05/63/PDF/Colloque_Lille_nov2006.pdf (02/09/2014).

DORMONT Brigitte, SAMSON Anne-Laure, 2009, Démographie médicale et carrières des médecins généralistes : les inégalités entre générations, Economie et Statistiques, ${ }^{\circ}{ }^{\circ} 414$, pp.3-30.

EDO Sophie, 2010, Etude comparative des conditions de vie et d'exercice des femmes médecins généralistes en milieu urbain, semi-urbain et rural en Isère. Thèse de doctorat en médecine présentée à l'Université Claude Bernard Lyon 1 [en ligne] http://www.urps-med-ra.fr/upload/ editor/These_EDO_Sophie_1326273406276.pdf (02/09/2014).

FENINA Annie, LE GARREC Marie-Anne, KOUBI Malik, 2010, Les comptes nationaux de la santé 2009, Drees Série Statistiques, Document de travail [en ligne] http://www.drees.sante.gouv.fr/ IMG/pdf/series tat149.pdf (02/09/2014).

GUILLUY Christophe, 2010. Fractures françaises, François Bourin Ed, 206 p. 
HAAS Sandrine, VIGNERON Emmanuel, 2010, La démographie médicale et paramédicale, L'inégalité des possibles, Gazette Des Communes, n 2047, Cahier détaché.

HARDY-DUBERNET Chantal, 2005, Femmes en médecine : vers un nouveau partage des professions?, Revue des Affaires sociales, La Documentation française, $\mathrm{n}^{\circ}$ 1, pp. 35-58.

HERIN Robert, 2003, Territoires de santé en Basse-Normandie, Géocarrefour, vol. 78/3, pp. 189-202.

HERITIER Françoise, 1996, Masculin, Féminin. La pensée de la différence, Paris : Ed. Odile Jacob.

JAKOUBOVITCH Steve, BOURNOT Marie-Christine, CERCIER Elodie, TUFFREAU François, 2012. Les emplois du temps des médecins généralistes, Etudes et Résultats, numéro 797, mars.

LAPEYRE Nathalie, 2006, Les professions face aux enjeux de la féminisation, Toulouse : Editions Octares, Coll. Travail et Activités humaines.

LAPEYRE Nathalie, LE FEUVRE Nicky, 2005, Féminisation du corps médical et dynamiques professionnelles dans le champ de la santé, Revue française des affaires sociales, $n^{\circ} 1$, pp. 59-81.

LE FEUVRE Nicky, 2001, La féminisation de la profession médicale : voie de recomposition ou de transformation du " genre »? In AIACH Pierre, CEBE Dominique, CRESSON Geneviève, PHILIPPE Claudine (dir.), 2001, Femmes et hommes dans le champ de la santé, approches sociologiques, Rennes : Presses de l'EHESP, Coll. Recherche Santé Social, pp. 197-228.

LE MAT Pacôme, BOURIN Stéphane, 2012, Médecin de campagne : une profession qui ne fait plus rêver [Reportage] In Journal télévisé France 3 Poitou-Charentes [en ligne] https:// www.youtube.com/watch?v=iYp9A941B70 (02/09/2014)

LEVY Danièle, 2011, Le métier de médecin aujourd'hui, Revue française des affaires sociales, $\mathrm{n}^{\circ}$ 2-3, pp. 297-309.

MAUREY Hervé, 2013, Déserts médicaux, agir vraiment, Rapport d'information de M. Hervé Maurey, fait au nom de la Commission du Développement durable, Sénat, $\mathrm{n}{ }^{\circ} 335$ (2012-2013), [en ligne] http://www.senat.fr/notice-rapport/2012/r12-335-notice.html (02/09/2014).

MOUILLOT Jérôme, 2013, 2 juillet, A Grandrieu, le débat sur le désert médical avance, Midi Libre, [en ligne] http://www.midilibre.fr/2013/07/01/a-grandrieu-le-debat-sur-le-desert-medicalavance,725426.php (02/09/2014).

NIEL Xavier, VILAIN Annick, 2001, Le temps de travail des médecins : l'impact des évolutions socio-démographiques, Drees Etudes et Résultats, $\mathrm{n}^{\circ} 114$, [en ligne] http:// www.drees.sante.gouv.fr/IMG/pdf/er114.pdf (02/09/2014).

OBSERVATOIRE POUR LA SECURITE DES MEDECINS (2013), Recensement national des incidents, IPSOS et Ordre National des Médecins [en ligne] http://www.conseil-national.medecin.fr/sites/ default/files/observatoire_securite_medecins_2013.pdf (02/09/2014)

PAICHELER Geneviève, 2001, Carrières et pratiques des femmes médecins en France (1930-1980) : portes ouvertes ou fermées? In AIACH Pierre, CEBE Dominique, CRESSON Geneviève, PHILIPPE Claudine (dir.), 2001, Femmes et hommes dans le champ de la santé, approches sociologiques, Rennes : Presses de l'EHESP, Coll. Recherche Santé Social, pp. 179-196.

PERROT Michelle, 1987, Qu'est-ce qu'un métier de femme ?, Le Mouvement social, $\mathrm{n}^{\circ}$ 140, pp. 2-10.

PERROT Michelle, 2002, Introduction In BARD Christine, CHAUVAUD Frédéric, PERROT Michelle, PETIT Jacques-Guy (dir.), Femmes et justice pénale, Rennes : Presses Universitaires de Rennes, pp. 125-128. 
PICHERAL Henri, 2001, Dictionnaire raisonné de géographie de la santé, Montpellier : Prennes de l'Université Montpellier III.

PIGEARD-MICAULT Natalie, 2011, La médecine : une profession féminine ? Une histoire de l'entrée des femmes en médecine In La médecine au féminin, Communication présentée lors de la Conférence à l'Université de Bordeaux Segalen « L'invité du mercredi/Saison 2010-2011, La médecine au féminin », 23 mars, Bordeaux [en ligne] http://www.canal-u.tv/video/ universite_bordeaux_segalen_dcam/

la_medecine_une_profession_feminine_une_histoire_de_l_entree_des_femmes_en_medecine. $6975(01 / 06 / 2014)$.

RAULT Jean-François, LE BRETON-LEROUVILLOIS Gwénaëlle, 2014, Atlas de la démographie médicale en France, Situation au 1er janvier 2014, Conseil National de l'Ordre des Médecins [en ligne] http://www.conseil-national.medecin.fr/sites/default/files/atlas_2014.pdf (02/09/2014).

SASSEN Saskia, 1996 (1ère ed 1991). La ville globale, Paris, Editions Descartes et Cie.

SCHWEITZER Sylvie, 2002, Les femmes ont toujours travaillé. Une histoire du travail des femmes, $\mathrm{XIX}^{\mathrm{e}}-\mathrm{XX}^{\mathrm{e}}$ siècle. Odile Jacob, Paris.

SICART Daniel, 2005, Les médecins, estimations au 1 er janvier 2005, Drees Séries statistiques, Document de travail [en ligne] http://www.drees.sante.gouv.fr/IMG/pdf/seriestat88.pdf (02/09/2014).

SICART Daniel, 2013, Les professions de santé au 1 er janvier 2013, Drees Séries Statistiques, Document de travail [en ligne] http://www.drees.sante.gouv.fr/IMG/pdf/seriestat179.pdf (02/09/2014).

SIMON Marc, NIEL Xavier, 1999, Les effectifs et la durée de travail des médecins au 1er janvier 1999, Drees Etudes et Résultats, $n^{\circ}$ 44, [en ligne] http://www.drees.sante.gouv.fr/IMG/ pdf/er044.pdf (02/09/2014).

TRONTO Joan, 1993. Moral boundaries. A political argument for an ethic of care, New YorkLondres, Routledge.

UNION REGIONALE DES MEDECINS LIBERAUX RHONE-ALPES, 2003, Etude de la féminisation de la profession médicale et de son impact. Approche quantitative et qualitative. Enquête auprès des médecins en exercice, CAREPS, [en ligne] http://www.urps-med-ra.fr/upload/urmlra/ urm_etude/pj/feminisation.pdf (02/09/2014).

ZOLESIO Emmanuelle, 2009, Des femmes dans un métier d'hommes : l'apprentissage de la chirurgie, Travail, Genre et Société, n ${ }^{\circ}$ 22, pp. 117-134.

\section{NOTES}

1. Le numerus clausus correspond à une limitation du nombre d'étudiants admis par concours en Faculté de Médecine depuis 1979.

2. La desserte médicale correspond au rapport entre la population totale (soit 63659608 habitants en France métropolitaine selon l'INSEE, Estimations provisoires

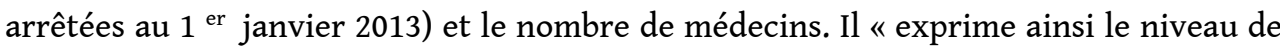
médicalisation d'une population dans un espace donné » (Picheral, 2001, p. 86). 
3. La densité médicale est le ratio qui rapporte les effectifs de médecins (omnipraticiens, spécialistes...) à la population d'un territoire donné. La densité médicale s'exprime en nombre de médecins pour 100,000 habitants.

4. Définition INSEE

5. Source: INSEE, Base Permanente des Equipements 2012 et INSEE Recensement Général de la Population 2010.

6. Source : RPPS au $1{ }^{\text {er }}$ janvier 2013, extraction ARS Bourgogne ; INSEE RGP 2010.

7. Les quartiers de Fontaine d'Ouche et des Grésilles à Dijon; du Mail à Chenôve; du Bief du Moulin à Longvic, du centre-ville à Quetigny et enfin du Belvédère à Talant.

8. Source: RPPS au 1 er janvier 2013, extraction ARS Bourgogne Et Observatoire Politique de la Ville du Grand Dijon.

9. Ce temps partiel (en moyenne de $33,9 \mathrm{~h}$ par semaine) se rapproche du temps de travail légal à temps plein $(35 \mathrm{~h})$, mais reste évidemment inférieur au taux horaire plein des médecins (de 52 à $60 \mathrm{~h}$ par semaine).

\section{RÉSUMÉS}

La féminisation de la profession médicale est un phénomène croissant, de plus en plus étudié par les historiens, sociologues ou médecins qui analysent son impact sur les évolutions du métier dans un contexte d'augmentation des inégalités en matière de santé. Mais alors que les inégalités de répartition des médecins vont croissantes, les géographes se sont peu penchés sur le lien entre féminisation et organisation territoriale de l'offre de soins. De nombreux rapports officiels et travaux scientifiques montrent que les écarts de répartition de médecins augmentent sur le territoire français et à différentes échelles (Maurey, 2013). La tendance actuelle est à la concentration des médecins en zone urbaine et par conséquent un délaissement des zones rurales désignées généralement par l'expression de " déserts médicaux ». L'objectif de cet article est d'explorer les facteurs couramment évoqués et perçus pour expliquer ce phénomène. Parmi ces derniers, nous verrons comment la féminisation de la profession médicale est souvent désignée comme un déterminant central des évolutions actuelles de la médecine et de la distribution inégale des médecins sur le territoire français. Outre la féminisation de la profession de médecins, il convient d'avoir à l'esprit que le secteur médical traverse également de nombreux changements liés au contexte actuel de départ massif à la retraite, à l'application du numerus clausus pendant de longues années et aux attentes de la nouvelle génération de médecins. Face à la diversité des facteurs démographiques, économiques, sociaux et territoriaux qui sont au cœur des changements de la profession, nous déterminerons la manière dont les représentations et les discours associés à la féminisation du métier perdurent et tendent à simplifier les évolutions complexes du métier.

The feminization of the physician workforce is a growing phenomenon, increasingly studied by historians, sociologists and physicians who examine the consequences of this shift on the medical profession in a context of increasing health inequalities. While inequality in health worker distribution is also increasing in France, geographers have little studied the relation between feminization and the territorial organization of healthcare provision. 
Many official reports and scientific studies show that disparities in the distribution of physicians increase over France and at different spatial levels (Maurey, 2013). Considering the workforces of physicians in relation to the French population, the situation of France is quite good but their distribution reveals clear spatial inequalities. Major disparities can be observed between administrative areas, for example "les régions". As shown in the map below, medical coverage is significantly higher in the French southern regions (particularly Provence-Alpes-Côte-d'Azur with one doctor for 244.77 inhabitants) and also the capital region called Ile-de-France (which one doctor for 252.22 inhabitants). On the contrary, the region called Picardie displays the lowest medical service (one doctor for 388.13 inhabitants) followed by the region Centre (one doctor for 375.43 inhabitants). Inequalities are even stronger within regions at a local level. Medical doctors concentrate mainly in urban areas and therefore neglect rural areas which appear and are described as "medical deserts". In 2012, 20\% of general physicians work in rural areas whereas $27 \%$ of French people live there.

The objective of this article is to explore the factors commonly discussed and perceived to explain this phenomenon. Among these, the feminization of the medical profession is often designated as a central determinant of the ongoing developments of the medical sector and the increasing spatial disparities among the French territory. We wanted to test the idea that medical deserts exist because of the feminization of the medical profession with local data, collected across the Burgundy region.

In Burgundy, the under-representation of female doctors in rural areas is real but the differences observed between the physical locations of male and female doctors are relatively moderate and not significantly different. However, some discourses from the medical sector, political sphere and media tend to associate closely the current increase of the spatial inequalities of medical care and the feminization of medical profession. The tweet from Senator Mayet "medical deserts, the fault of the women?" of 27 July 2015 can certainly appear anecdotal but it still shows an overview of current thinking that can circulate on the effects of the feminization of the medical workforce.

To understand the rationales behind medical deserts, it appeared that we should go beyond gendered explanations in order to address the broader social and economic determinants which contribute to this phenomenon. Some recent studies show that young medical practitioners, both women and men, wish to preserve time for family or for social activities leading them to reconsider their relationships to work (Deriaz et al. 2010). In 2003, Robert Herin already stated about the liberal healthcare provision in Normandy that the new generations of physicians (regardless of gender) were seriously concerned by living environment, educating children, the range of jobs for spouses, the qualities of cultural and social environment, etc. All these determinants tend therefore to lead young doctors towards urban environments more than rural areas. As a consequence, we must consider the spatial disparities observed among the medical physicians in France more as a generational issue than a simple gendered issue.

INDEX

Mots-clés : pratiques professionelles, genre, féminisation, génération

\section{AUTEURS}

\section{ALICE DENOYEL-JAUMARD}

Géographe - UMR CNRS 5600 Environnement, Ville, SociétéCentre de Recherche en GéographieAménagement - Université Jean Moulin-Lyon 3 


\section{AUDREY BOCHATON}

Maître de conférences en géographie, Université Paris Ouest Nanterre la Défense UMR 7533

Laboratoire Dynamiques Sociales et Recompositions des Espaces 